\title{
Collective patterns arising out of spatio-temporal chaos
}

\author{
Shin-ichi Sasa \\ Department of Pure and Applied Sciences, College of Arts and Sciences, University of Tokyo, \\ Komaba, Meguro-ku, Tokyo 153, Japan
}

(November 10, 2018)

\begin{abstract}
We present a simple mathematical model in which a time averaged pattern emerges out of spatio-temporal chaos as a result of the collective action of chaotic fluctuations. Our evolution equation possesses spatial translational symmetry under a periodic boundary condition. Thus the spatial inhomogeneity of the statistical state arises through a spontaneous symmetry breaking. The transition from a state of homogeneous spatio-temporal chaos to one exhibiting spatial order is explained by introducing a collective viscosity which relates the averaged pattern with a correlation of the fluctuations.

05.45.+b,47.52. $+\mathrm{j}, 47.54+\mathrm{r}$
\end{abstract}

Typeset using REVTEX 
Macroscopic structures formed in turbulent flow, such as roll patterns seen in clouds and the red spot on Jupiter, are often observed in nature. These phenomena have been explained using a plausible argument that an equation describing mean flow is renormalized by turbulent fluctuations and displays an instability under certain conditions. Much progress has been made toward justifying this explanation [1].2], but there has been little study of the origin of the mean flow. If a system possesses spatial inhomogeneity, it is easily understood how mean flow can arise, but this is not the case in which we are interested. In this paper, we consider the emergence of mean flow through a spontaneous symmetry breaking of the statistical state in turbulence.

Several recent experiments seem to show the formation of a mean flow in turbulence. In thermo-convective systems, two different types of turbulence, soft and hard turbulence, can be observed, depending on the Rayleigh number, and it has been found that hard turbulence is characterized by the existence of mean flow [3]. Because the experiments in question have been done on systems with small aspect ratios, effects of horizontal boundaries cannot be neglected, and the spatial translational symmetry is broken externally. However, the systems do possess discrete symmetry associated with a spatial parity. The mean flow then breaks the parity symmetry spontaneously, that is, the direction of the large scale circulation flow is determined by the initial conditions. We note that according to recent numerical simulations of the Boussinesq equation [4], the horizontal boundaries do not play an essential role in the formation of mean flow. We thus expect that mean flow may occur even in the infinite limit of the aspect ratio. Actually, the emergence of mean flow in convective turbulence in a system with large aspect ratio (about 1000) has been found in the electro-hydrodynamic convection of nematics [5], where persistent roll patterns appear in the turbulent state. In this case, the mean flow can be thought to spontaneously break the spatial translational symmetry in turbulence. Additionally, it has been reported recently that chaotic wave patterns produced by the Faraday instability display spatially ordered time averages [6]. Similar phenomena have also been discovered in Taylor-Coutte flow [7] and rotating convection [8].

These experimental results suggest that mean flow and persistent patterns can appear 
spontaneously in various chaotic systems. We propose to call these 'collective patterns in spatio-temporal chaos', because their emergence results from collective action of turbulent fluctuations, as explained below. This phenomenon should be considered together with collective motion in high-dimensional chaos, which has been discovered in spatially extended systems [9, 10] and globally coupled systems [11, [12]. We believe that the collective patterns we consider presently are closely related to these types of collective motion.

In this paper, we would like to focus on the universal aspects of the phenomena in question, not system dependent details. We first present a simple mathematical model which exhibits the emergence of collective patterns out of spatio-temporal chaos. We then explore how a transition occurs from a state of homogeneous spatio-temporal chaos to one displaying spatial order by analyzing this model.

Equations of motion in fluid systems are written as

$$
\partial_{t} q^{(i)}+\vec{\nabla} \cdot \vec{J}^{(i)}=0
$$

where $q=\left(q^{(1)}, \cdots, q^{(M)}\right)$ is an $M$-component field defined in a $D$-dimensional space, and the current $\vec{J}=\left(\vec{J}^{(1)}, \cdots, \vec{J}^{(M)}\right)$ is a functional of the field $q$, (e.g. $q$ is given as $q=(\vec{v}, T)$ in thermo convective systems.) Eq.(11) is often referred to as a continuity equation associated with some conservation law. We note, however, that $Q^{(i)}=\int d x^{D} q^{(i)}$ is not a conserved quantity in a finite system where the surface integral of the current does not vanish. Nevertheless, when we focus on a bulk region in a turbulent system whose fluctuations develop only to a length scale much smaller than the system size, we can regard $Q^{(i)}$ as a nearly conserved quantity. This property plays an essential role in the formation of collective patterns, because large scale dynamics of conserved quantities determine the long-time behavior of fluctuations. Further, due to the fact that the wavelength of collective roll patterns is of the same order as the role height in convective systems, we conjecture that the formation of collective roll patterns is related to a breakdown of conservation laws. Based on these considerations, we propose the following one-dimensional model equation in order to study collective patterns: 


$$
\partial_{t} q^{(i)}+\partial_{x} J^{(i)}=\epsilon^{2} F^{(i)},
$$

where $F=\left(F^{(1)}, \cdots, F^{(M)}\right)$ is a functional of the field $q$, and $\epsilon$ is a small parameter which measures the extent of the breakdown of the conservation properties. The system size $L$ is assumed to be much larger than the correlation length of fluctuations in spatio-temporal chaos, and a periodic boundary condition is assumed so as to maintain spatial translational symmetry.

The simplest equation exhibiting spatio-temporal chaos with a conserved quantity is the Kuramoto-Sivashinsky (KS) equation,

$$
\partial_{t} v+\partial_{x}\left(v^{2}+\partial_{x} v+\partial_{x}^{3} v\right)=0,
$$

which has been derived in several contexts, including chemical waves, front propagation, etc. [13]. Statistical properties of turbulent solutions of the KS equation are homogeneous under periodic boundary conditions and have been investigated extensively [14]. Although it was found that the homogeneity of the statistical state is broken under a rigid boundary condition [15], we do not consider such an external symmetry breaking. Keeping in mind the guiding principle of modeling mentioned above, we studied a modified KS equation supplemented with a small term which breaks a conservation law. However, as far as we have checked, statistical properties seem to remain homogeneous if $F$ is restricted to a local functional of $v$. Proceeding to the next step, we considered a two component system (i.e. $M=2$ ) in which a second field is coupled to the KS equation, and found that the dynamical behavior is drastically changed. The model equation we discuss in this paper takes the form

$$
\begin{aligned}
\partial_{t} v+\partial_{x}\left(v^{2}+\partial_{x} v+\partial_{x}^{3} v\right) & =-b v-d u, \\
\partial_{t} u-D \partial_{x}^{2} u & =a u+c v-g u^{3},
\end{aligned}
$$

where $a, b, c, d$ and $g$ are assumed to be small positive parameters proportional to $\epsilon^{2}$. These values are chosen so that the trivial solution $u=v=0$ is stable in the absence of the term $\partial_{x}^{2} v$, which produces spatio-temporal chaos. The condition for this stability is found to be 
$b>a$ and $-a b+c d>0$, and we therefore assume throughout this paper that $a=\epsilon^{2}, b=2 \epsilon^{2}$, $c=\epsilon^{2}, d=3 \epsilon^{2}$, and $g=\epsilon^{2}$.

Now, we present results of our numerical simulations of Eq.(田), where we have used a simple discretization scheme with a spatial mesh size $\delta x=1.0$ and time step size $\delta t=0.1$. This crude numerical scheme is sufficient for our purposes. (We note that our numerical scheme preserves a discrete spatial translational symmetry.) The values of $\epsilon$ and $L$ were varied between 0.1 and 0.025 and between 256 and 2048, respectively, because we are interested in the system behavior under the asymptotic condition $(\epsilon, L) \rightarrow(0, \infty)$. The diffusion constant $D$ is treated as a control parameter. In Fig. 1, spatio-temporal patterns are displayed for different values of $D$, with $(\epsilon, L)=(0.1,256)$. In panel (a), small scale fluctuations are seen as usual in spatio-temporal chaos, while in panel (b), we can find that "a coarsegrained periodic pattern" is superimposed on the small scale fluctuations. This suggests that our model can describe a transition to spatio-temporal chaos with a collective pattern. We confirmed quantitatively the existence of the average pattern by measuring the quantity $<v(x)>$, where we have introduced the abbreviated notation

$$
<f>=\frac{1}{T} \int_{T_{0}}^{T_{0}+T} d t f(t) .
$$

Here, $T_{0}$ is some initial, and $T$ is the averaging time. In order to check whether or not the field $\langle v(x)>$ has a spatial variation in the limit $T \rightarrow \infty$, we measured the quantity

$$
\sigma^{2}=\frac{1}{L} \int d x\left|<v(x)>-\frac{1}{L} \int d x<v(x)>\right|^{2},
$$

where the spatio-temporal chaos is considered to be homogeneous when the scaling relation $\sigma \sim 1 / \sqrt{T}$ is obtained for sufficiently large $T$. Figure 2 shows the $T$ dependence of $\sigma$ for systems in which $(\epsilon, L)=(0.1,1024)$. $\sigma$ decays to 0 as $\sigma \sim 1 / \sqrt{T}$ when $D=1.5$, while it seems to keep a constant value when $D=0.5$. We thus expect that there is a critical value $D_{c}$ between 0.5 and 1.5. The $D$ dependence of $\sigma$ for a sufficiently large $T(T=12800)$, the phase diagram for our system, is shown in Fig. 3. This graph suggests that $D_{c}$ is near 0.8.

We now attempt to explain how the collective patterns emerge out of spatio-temporal chaos. First, let us derive a self-consistent condition under which a long time averaged 
quantity $\langle v(x)>$ forms a periodic pattern. For simplicity, we focus on the asymptotic case that $(\epsilon, L) \rightarrow(0, \infty)$ and suppose the $\epsilon$ dependences of $\langle u\rangle$ and $\langle v\rangle$ can be expressed as $\langle u\rangle=U(\epsilon x)$, and $\langle v\rangle=V(\epsilon x)$. These scaling forms are consistent with numerical results. Then, substituting $u=\left\langle u>+u^{\prime}\right.$ and $v=\left\langle v>+v^{\prime}\right.$ into Eq.(4), averaging the equation, and discarding $o\left(\epsilon^{2}\right)$ terms in the equation, we obtain

$$
\begin{array}{r}
\partial_{x}\left(V^{2}+<v^{\prime 2}>+\partial_{x} V\right)=-b V-d V \\
-D \partial_{x}^{2} U=a U+c V-g U^{3}
\end{array}
$$

where we have assumed $\left\langle(u-\langle u\rangle)^{k}\right\rangle=o(\epsilon)$ with $k=2,3$. Now, in order to obtain a closed form of the equation for $U$ and $V$, we employ the phenomenological relation

$$
<v^{\prime 2}>=\alpha-\nu \partial_{x}<v>
$$

which has been used to analyze turbulent shear flow [2]. Here, $\alpha$ and $\nu$ are assumed to be constants, and $\nu$ is often referred to as "turbulent viscosity". We can see from Eqs.(7) and (8) that $\nu$ expresses how turbulent fluctuations contribute a dissipation effect on the coarse-grained pattern. Although the validity of Eq.(8) for our equation is not proved mathematically, as shown in Fig. 止, our numerical results for $\left\langle v^{\prime 2}>\right.$ and $\langle v\rangle$ are consistent with this relation. Further, under a mode truncation approximation, we write $<v>=R \sin (k x+\phi)$ and $\langle u>=S \sin (k x+\phi)$, and obtain the equation for $R$ and $S$

$$
\begin{aligned}
\left(D_{*} k^{2}+b\right) R+d S & =0, \\
-c R+\left(D k^{2}-a\right) S & =-g S^{3},
\end{aligned}
$$

where $k$ is of order $\epsilon$, in accordance with the above stated assumption, and $D_{*}=\nu-$ 1. Equation (9) always has the trivial solution $R=S=0$, which represents a spatially homogeneous statistical state, while a non-trivial solution exists only when the inequality

$$
D<\frac{2-\sqrt{3}}{2} D_{*}
$$

is satisfied. This result is consistent with our phase diagram shown in Fig.3. In fact, by fitting our numerical data to Eq.(8), we found $\nu \sim 5.5$ in the case that $(D, \epsilon, L)=(0.2,0.1,1024)$. 
Using this value of $\nu$ for all $D$, we obtain $D_{c} \sim 0.6$. The discrepancy between this value and that obtained from our phase diagram in Fig. 3 may be due to the fact that $\nu$ depends on $(D, \epsilon, L)$.

The self-consistent approach developed above can tell us nothing about the stability of the obtained solutions. In order to consider the stability problem, we need to develop a theory describing the slow evolution of coarse-grained patterns. A derivation of the evolution equation from our model equation is beyond the scope of this paper, but we present the results we obtained using the scaling relation such that $v(x, t)=V\left(\epsilon x, \epsilon^{2} t\right)+v^{\prime}(x, t)$. We found that the homogeneous statistical state becomes unstable when the inequality (10) is satisfied, and in this case, a coarse-grained periodic pattern with a wavenumber $q_{m}$ grows with the maximum growth rate, where

$$
q_{m}=\epsilon \sqrt{\frac{D_{*}-2 D}{2 D_{*} D}} .
$$

That is, a non-trivial persistent pattern appears when the homogeneous statistical state becomes unstable. This statement is consistent with our numerical experiments. Here, we would like to stress that $D_{c}$ and $q_{m}$ are determined by $D_{*}$, which is not a parameter of our model but associated with correlations of fluctuations. Therefore, the existence of the averaged pattern cannot be explained by developing a linear stability analysis of the trivial state $u=v=0$.

As argued above, the collective action of fluctuations causes an instability leading to the formation of persistent patterns. In this sense, we can say that fluctuations are the origin of a spatial order. On the other hand, one may suspect that fluctuations act as a turbulent noise, inducing a random drift of our coarse-grained pattern, like Brownian motion, and as a result the averaged pattern vanishes in the limit that the averaging time $T$ goes to infinity. In order to check this, we calculated a time series of the complex amplitude of the Fourier mode associated with the collective pattern, $\{A(t) \exp (i \phi(t))\}$. Spectra of the amplitude $A$ and phase $\phi$, which are denoted by $S_{A}(\omega)$ and $S_{\phi}(\omega)$ respectively, are shown in Fig. 5. The spectrum $S_{\phi}(\omega)$ goes as $\omega^{-2}$ at low frequency $\left(\omega<\omega_{c}\right)$. This implies the existence of a phase 
diffusion of the coarse-grained pattern and therefore that the averaged pattern will vanish when the averaging time $T$ is chosen much larger than $2 \pi / \omega_{c}$. We should note, however, that the crossover frequency $\omega_{c}$ is much smaller than the inverse of the correlation time of chaotic fluctuations, and from our preliminary numerical results we believe that $\omega_{c} \rightarrow 0$ for $\epsilon \rightarrow 0$, with $\epsilon L$ fixed. This implys that if the limit $T \rightarrow \infty$ is considered after taking $\epsilon \rightarrow 0$ with fixed $\epsilon L \geq O(1)$, persistent patterns actually exist. Figure 5 shows further that the amplitude $R$ has a long-range correlation. At low frequency $\left(\omega<\omega_{c}\right)$, the form of $S_{R}(\omega)$ seems to be a Lorenzian type, proportional to $\left(1+(\omega \tau)^{2}\right)^{-1}$. Here, the correlation time of the amplitude fluctuation, $\tau$, which may depend on $(\epsilon, L)$, is much larger than $2 \pi / \omega_{c}$. Although we believe that $\tau \omega_{c} \rightarrow \infty$ for $\epsilon \rightarrow 0$ with fixed $\epsilon L$, it is necessary to study the $(\epsilon, L)$ dependence of the system behavior further.

In conclusion, we have shown that our model equation can exhibit the emergence of collective patterns out of spatio-temporal chaos. We believe that such a phenomenon will be observed in other mathematical models which have nearly conserved quantities. By introducing a small parameter $\epsilon$ which measures the extent of the breakdown of conservation properties, we have developed a self-consistent analysis which describes a transition to the spatially ordered phase. Both fluctuations of the amplitude and phase of the Fourier mode associated with the collective pattern have long range correlations at a time scale larger than $2 \pi / \omega_{c}$, while over a time scale $2 \pi / \omega_{c}$, which goes to infinity for $\epsilon \rightarrow 0$, averaged patterns are properly defined. In our theoretical analysis, the relation between the averaged pattern and the correlation of fluctuations around it plays a crucial role. Although the relation (8) is a familiar one at a phenomenological level, there may be various forms which relate the statistical property of fluctuations with an averaged quantity. We expect that as yet unknown relations will be discovered in experimental systems and other mathematical models. Also, to determine the correspondence between our model and experimental systems may be an important subject. Such studies should contribute greatly to the understanding of the nature of spatio-temporal chaos.

I am grateful to M. Sano for active exchange of ideas concerning his experiment and 
the present study. I thank Y-h. Taguchi and Y. Iba for their critical comments. I also thank Y. Kuramoto, S. Toh, S. Nasuno, G.C. Paquette, K. Kaneko and S. Kai for fruitful discussions. H. Chaté, P. Marcq and P. Manneville are acknowledged for their hospitality and stimulating discussions during my three week stay at Saclay in the early stage of this study. 


\section{REFERENCES}

[1] B. Dubrulle nd U. Frisch, Phys.Rev A43, 5355, (1991).

[2] D. J. Tritton, Physical Fluid Dynamics, (Clarendon press, Oxford, 1988).

[3] F. Heslot, B. Castaing, and A. Libchaber, Phys.Rev A36, 5870, (1987); X.Z. Wu, L. Kaddanoff, A. Libchaber, and M. Sano, Phys.Rev.Lett 64, 2140, (1990).

[4] S. Toh, private commnication.

[5] M. Sano, private communication; H. Yamazaki, K. Hirakawa and S. Kai, Mol. Cryst. Liq.Cryst.122, 41, (1985).

[6] B.J. Gluckman, P. Marcq, J. Bridger, and J.P. Gollub, Phys.Rev.Lett 71, 2034, (1993); B.J. Gluckman, C.B. Arnold and J.P. Gollub, Phys.Rev. E 51, 1128, (1995).

[7] D. P. Lathrop, J. Fineberg, and Harry L. Swinny, Phys.Rev.Lett 68 1515, (1992).

[8] L. Ning, Y. Hu, R.E. Ecke, and G. Ahlers, Phys.Rev.Lett 71, 2216, (1993).

[9] H. Chaté and P. Manneville, Prog.Theor.Phys. 88, 1, (1992); H. Chaté and P. Manneville, Europhys.Lett. 14, 409, (1992); H. Chaté and P. Manneville, Europhys.Lett. 17, 291, (1992); L. Brunnet, H. Chaté, and P. Manneville, Physica D 78, 141, (1994 ).

[10] T. Bohr, G. Grinstein, Yu He, and C. Jayaprakash, Phys.Rev.Lett, 58, 2155, (1987).

[11] K. Kaneko,Phys. Rev. Lett. 65, 1391 (1990); K. Kaneko,Physica D 55, 368 (1992); A.S. Pikovsky and J. Kurths, Phys.Rev.Lett. 72, 1644,(1994).

[12] N. Nakagawa and Y. Kuramoto, Physica D 80, 307, (1995).

[13] Y. Kuramoto, Chemical Oscillations, Waves, and Turbulence, (Springer, Berlin Heidelberg, 1984); Y. Kuramoto and T. Tsuzuki, Prog.Theor.Phys. 55, 356, (1976).

[14] K. Sneppen, J. Krug, M.H. Jensen, C. Jayaprakash, and T. Bohr, Phys.Rev. A46, R7351, (1992).

[15] S. Zaleski and P. Lallemand, J. Physique Lett. 46, L793, (1985). 


\section{FIGURES}

FIG. 1. Spatio-temporal patterns of the $v$ field of our model equation with $(\epsilon, L)=(0.1,256)$. We have, (a) $D=0.5$ and (b) $D=1.5$. The time goes from $t=10000$ to $t=22800$.

FIG. 2. $\sigma$ versus $T$ for $D=0.5$ (solid line) and $D=1.5$ (dashed line) $(\epsilon, L)=(0.1,1024)$. The transient time was chosen as $T_{0}=10000$.

FIG. 3. $\sigma$ versus $D$ for $T_{0}=10000$ and $T=12800 .(\epsilon, L)$ is as in Fig. 2. Initial conditions are the same for all values of $D$.

FIG. 4. Snapshot of the $v$ field at $t=505000$ (dotted line), the averaged pattern (solid line), and the dispersion at each point (dotted line), for the system with $(D, \epsilon, L)=(0.2,0.1,1024)$. Each field in the region $0<x<256$ is displayed. The statistical procedure was carried out for a sample $\{v(x, t) ; 5000<t<505000\}$.

FIG. 5. Spectra for a time series of the amplitude (solid line) and phase (dotted line) of a Fourier mode $\exp (i k x)$ associated with a collective pattern with the same parameter values as in Fig. 4. The value of $k$ is given as the position of the peak of the spectrum of the averaged pattern. These graphs were obtained by averaging 8 samples of the spectra using 8192 data points every 128 time units. 


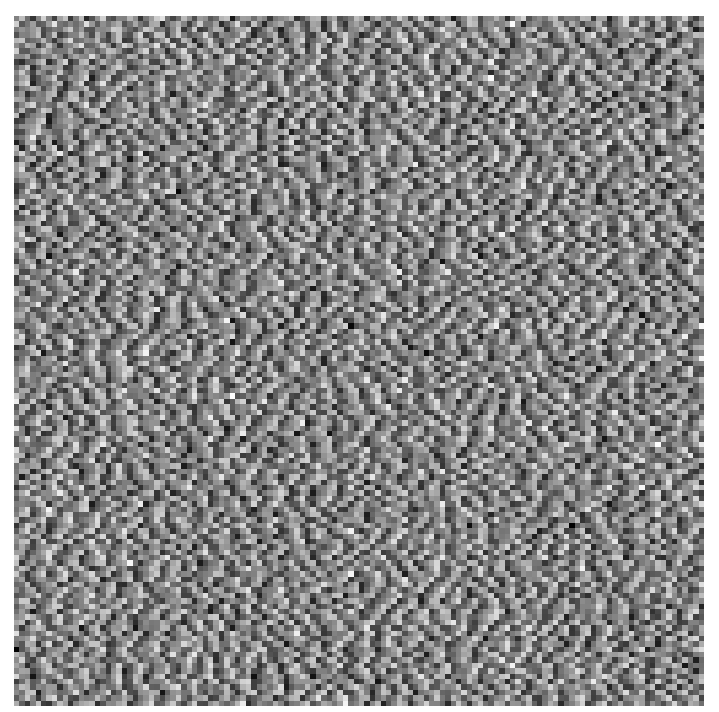

Fig. 1a S. Sasa 


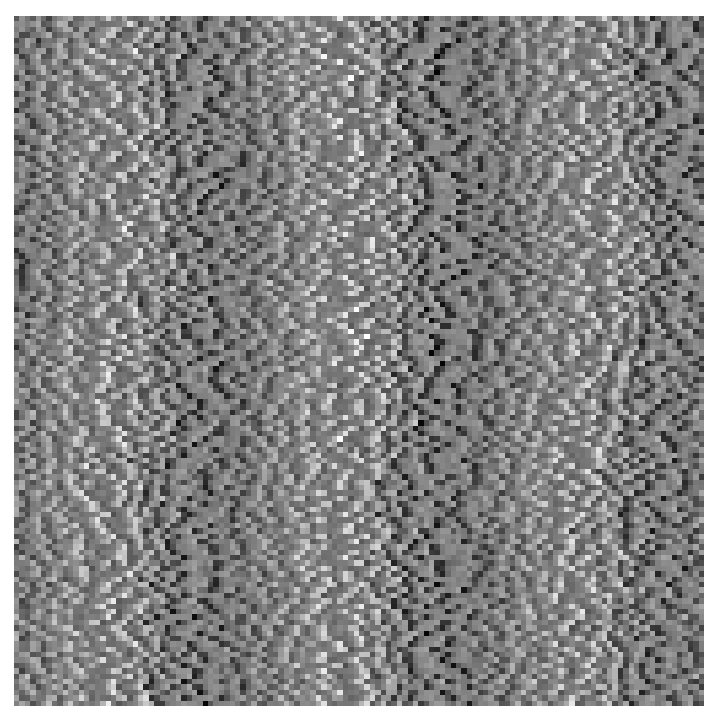

Fig.1b S. Sasa 


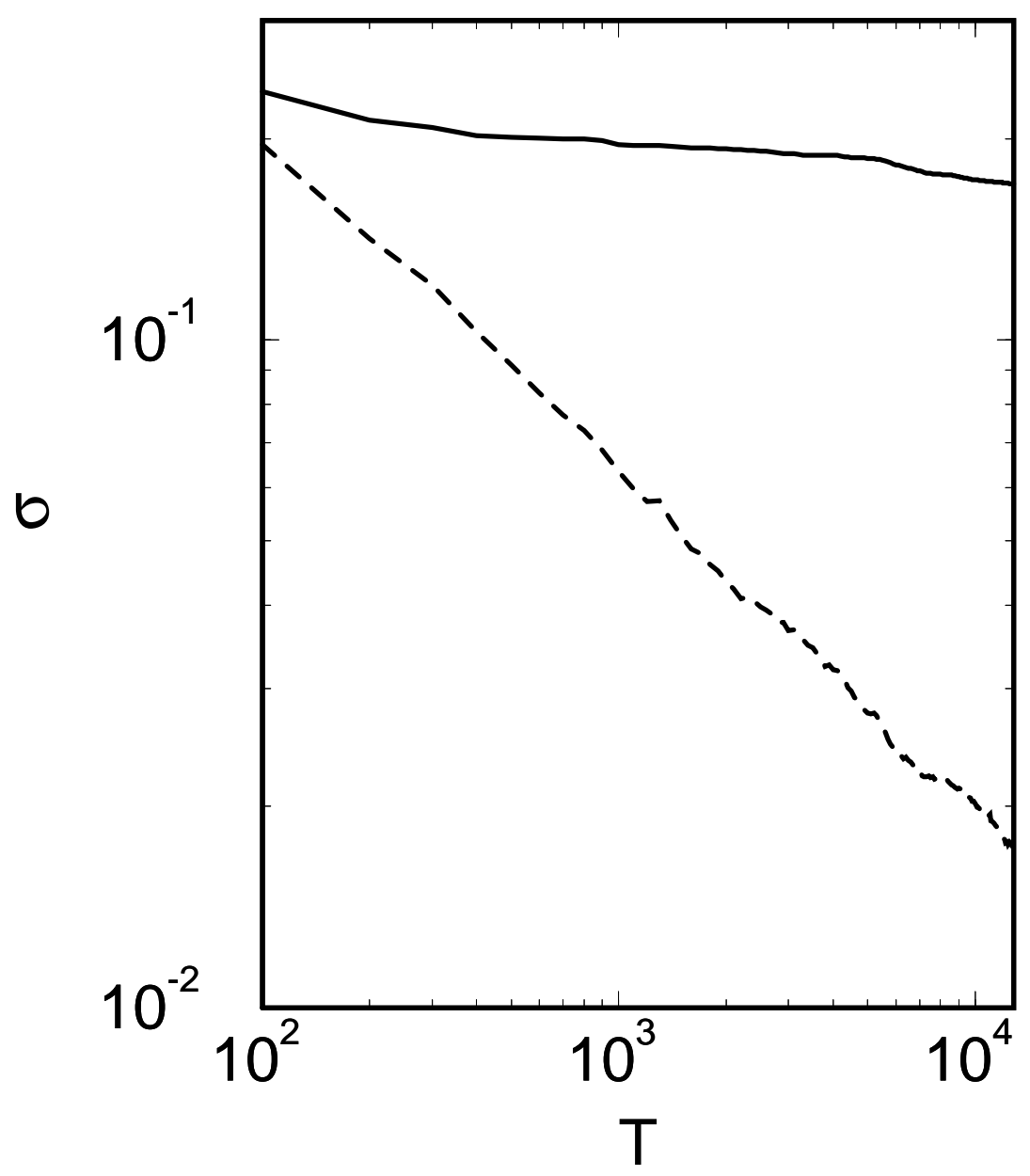

Fig.2 S. Sasa 


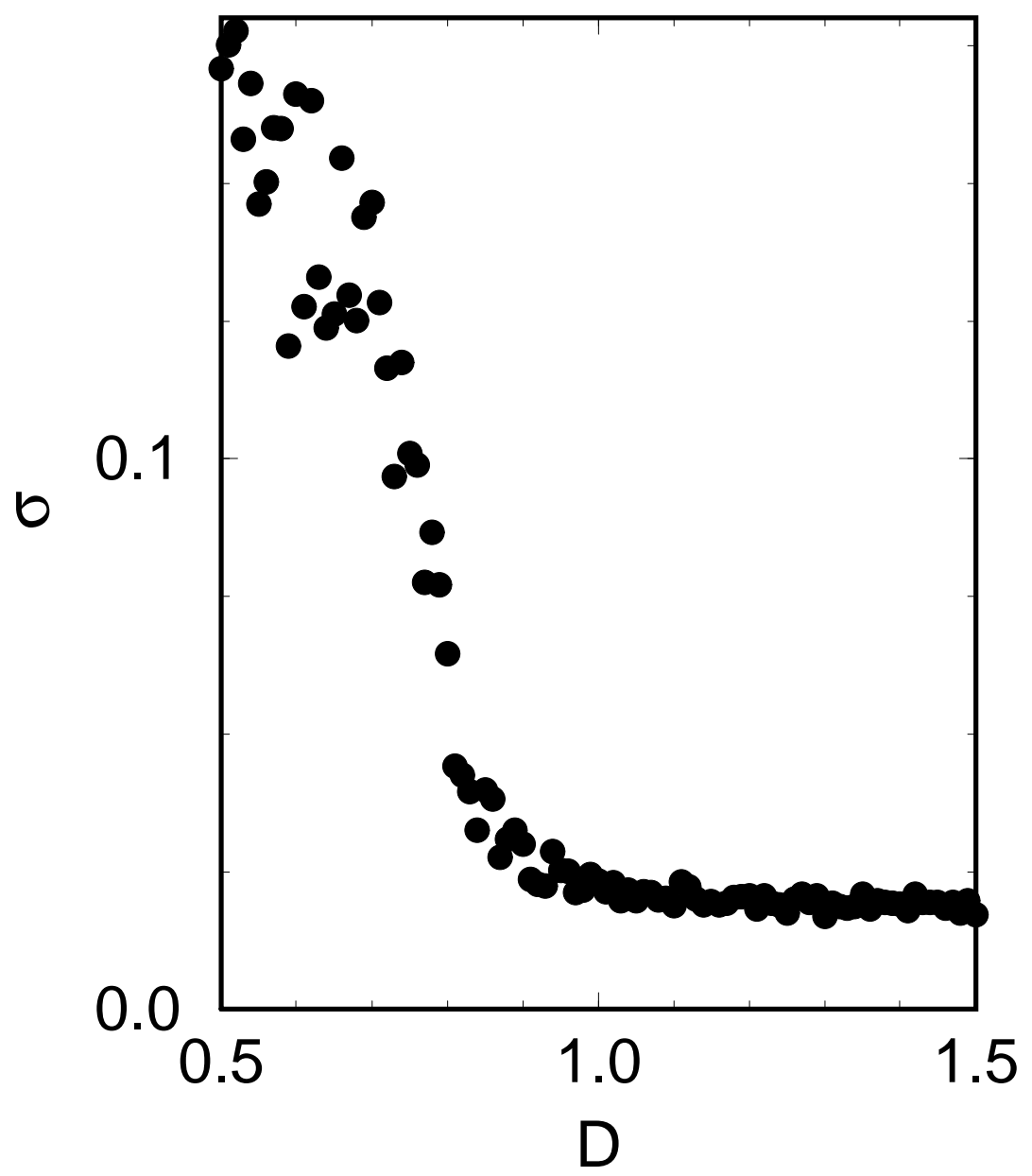

Fig.3 S.Sasa 


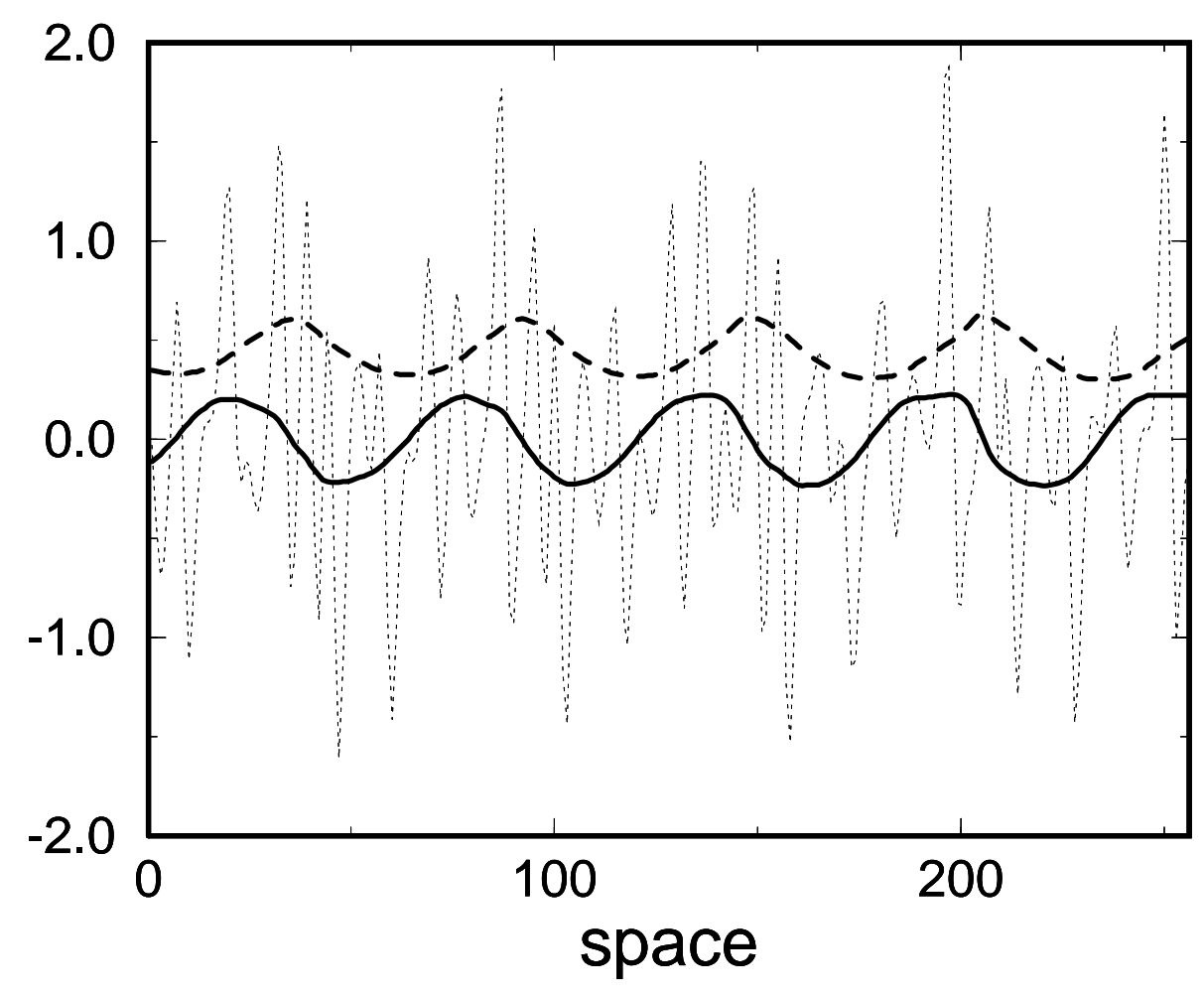

Fig.4 S.Sasa 


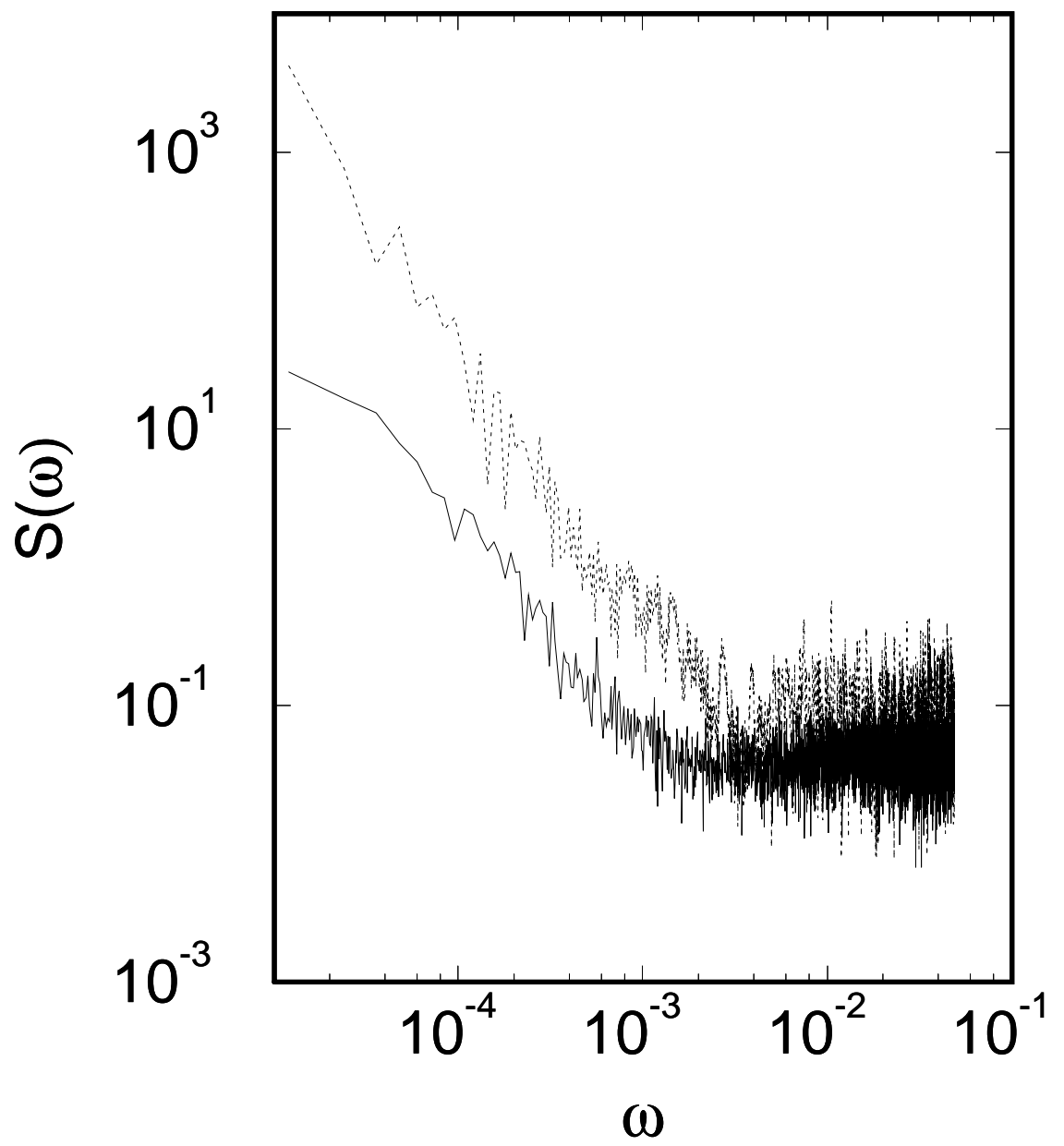

Fig. 5 S.Sasa 